\title{
Certain Subclasses of Analytic and Bi-Univalent Functions Involving Double Zeta Functions
}

\author{
Saibah Siregar, Sintuja Raman \\ Faculty of Science and Biotechnology, University Selangor, Bestari Jaya, Selangor Darul Ehsan, 45600, Malaysia \\ E-mail:saibahmath@yahoo.com,thuja_88@yahoo.com
}

\begin{abstract}
In the present paper, we introduce two new subclasses of the functions class $\Sigma$ of bi-univalent functions involving double zeta functions in the open unit disc $U=\{z: z \in \mathbb{C},|z|<1\}$. The estimates on the coefficients $\left|a_{2}\right|$ and $\left|a_{3}\right|$ for functions in these new subclasses of the function class $\Sigma$ are obtained in our investigation.
\end{abstract}

Keywords - Analytic functions, Univalent functions, Bi-univalent functions, Starlike and convex function, Coefficients bounds.

\section{INTRODUCTION}

Let $A$ be the class of the function of the form

$$
f(z)=z+\sum_{k=2}^{\infty} a_{k} z^{k}
$$

which are analytic in the open unit $\operatorname{disc} U=\{z:|z|<1\}$. Further, by $S$ we shall denote the class of all functions in $A$ which are univalent in $U$. By using the Hadamard product or the convolution product of generalized Hurwitz-Lerch zeta function given by [4], a function is defined as follows:

$$
\Psi_{n}(y, x, a)=\frac{\Phi(y, x, a+v n)}{\Phi(y, x, a)}
$$

It is clear that $\Psi_{0}(y, x, a)=1$. Now consider the function

$$
\Upsilon_{\mu}(z, y, x, a)=\sum_{n=0}^{\infty} \frac{\mu_{n}}{n !} \Psi_{n}(y, x, a) z^{n}
$$

implies

$$
z \Upsilon_{\mu}(z, y, x, a)=z+\sum_{n=2}^{\infty} \frac{(\mu)_{n-1}}{(n-1) !} \Psi_{n-1}(y, x, a) z^{n}
$$

Thus

$$
\begin{aligned}
\Upsilon_{\mu}(z, y, x, a) *\left(z \Upsilon_{\mu}(z, y, x, a)\right)^{-1} & =\frac{z}{(1-z)^{\lambda}}, \quad(\lambda>-1) \\
& =z+\sum_{n=2}^{\infty} \frac{(\lambda)_{n-1}}{(n-1) !} z^{n}
\end{aligned}
$$

poses a linear operator

$$
I_{\mu}^{\lambda}(z, y, x, a) f(z)=\left(z \Upsilon_{\mu}(z, y, x, a)\right)^{-1} * f(z),(f \in A)
$$

$$
=z+\sum_{n=0}^{\infty} \frac{(\lambda)_{n-1}}{(\mu)_{n-1} \Psi_{n-1}(y, x, a)} a_{n} z^{n}
$$

where $|y|<1,|z|<1 ; \mu \in \mathbb{C}\{\ldots-2,-1,0\}, v \in \mathbb{C}\{0\}$;

$a \in \mathbb{C}\{-(m+v n)\},\{n m\} \in \mathbb{N} \cup\{0\}$ and $\Psi$ is defined in (2). It is clear that $I_{\mu}^{\lambda}(z, y, x, a) f(z) \in A$. It is based on result by Ibrahim and Darus.

It is well known that every function $f \in S$ has inverse $f^{-1}$, defined by $f^{-1}(f(z))=z \quad(z \in U)$ and

$$
f\left(f^{-1}(w)\right)=w \quad\left(|w|<r_{o}(f) \geq \frac{1}{4}\right)
$$

where

$$
f^{-1}=w-a_{2} w^{2}+\left(a_{2}^{2}-a_{3}\right) w^{3}-\left(5 a_{2}^{3}-5 a_{2} a_{3}+a_{4}\right) w^{4}+\ldots .
$$

A function $(f \in A)$ is said to be bi-univalent in $U$ if both $f(z)$ and $f^{-1}(z)$ are univalent in $U$. Let $\sum$ denote the class of bi-univalent in $U$ given by the Taylor-Maclaurin series expansion (1). Examples of functions in the class $\Sigma$ are

$$
\frac{z}{(1-z)},-\log (1-z), \frac{1}{2} \log \left(\frac{1+z}{1-z}\right),
$$

and so on. However, the familiar Koebe function is not a member of $\sum$. Other common examples of functions in $S$ such as $z-\frac{z^{2}}{2}$ and $\frac{z}{1-z^{2}}$ are also not members of $\Sigma$.

Lewin [4] investigated the bi-univalent function class $\Sigma$ and showed that $\left|a_{2}\right|<1.51$. Subsequently, Brannan and 
Clunie [5] conjectured that $\left|a_{2}\right| \leq \sqrt{2}$. Netanyahu [6], on the other hand, showed that $\max _{f \in \Sigma}\left|a_{2}\right|=\frac{4}{3}$.

The coefficient estimate problem for each of the following Taylor-Maclaurin coefficients:

$\left|a_{n}\right| \quad(n \in \mathbb{N} \backslash\{1,2\} ; \mathbb{N}:=\{1,2,3, \ldots\})$ is presumably still an open problem.

Brannan and Taha [7] (see also [8]) introduced certain subclasses of the bi-univalent function class $\sum$ similar to the familiar subclasses $S_{*}(\kappa)$ and $K(\kappa)$ are starlike and convex function of order $\kappa,(0 \leq \kappa<1)$, respectively (see[9]). Thus, following Brannan and Taha [7] (see also [8]), a function $f \in A$ is in the class $S_{\Sigma}^{*}(\alpha)(0<\alpha \leq 1)$ of strongly bi-starlike functions of order $\alpha$ if each of the following conditions is satisfied:

$$
f \in \sum \text { and }\left|\arg \left(\frac{z f^{\prime}(z)}{f(z)}\right)\right|<\frac{\alpha \pi}{2},
$$

$(z \in U ; 0<\alpha \leq 1)$ and

$$
\left|\arg \left(\frac{w g^{\prime}(w)}{g(w)}\right)\right|<\frac{\alpha \pi}{2},(w \in U ; 0<\alpha \leq 1)
$$

where $g$ is the extension of $f^{-1}$ to $U$. The classes $S_{\Sigma}^{*}(\kappa)$ and $K_{\Sigma}(\kappa)$ of bi-starlike functions of order and bi-convex functions of order $K_{i}$, corresponding (respectively) to the function classes $S_{*}(\kappa)$ and $K(\kappa)$ were also introduced analogously. For each of the function classes $S_{\Sigma}^{*}(\kappa)$ and $K_{\Sigma}(\kappa)$, they found non-sharp estimates on the first two Taylor-Maclaurin coefficients $\left|a_{2}\right|$ and $\left|a_{3}\right|$ (for details, see $[7,8])$.

The object of the present paper is to introduce two new subclasses of the functions class $\sum$ involving double zeta functions operator and find estimates of the coefficients $\left|a_{2}\right|$ and $\left|a_{3}\right|$ for functions in these new subclasses of the function class $\sum$. The techniques of proofing used by Srivastava et. al [4].

\section{COEFFICIENT BOUNDS FOR THE FUNCTION CLASS $\mathscr{H}_{\Sigma}^{\alpha}$}

Definition 1. A function $\mathrm{f}(\mathrm{z})$ given by (1.1) is said to be in the class $\mathscr{H}_{\Sigma}^{\alpha}(0<\alpha \leq 1)$ if the following conditions are satisfied: $f \in \sum$ and

$$
\left|\arg \left(I_{\mu}^{\lambda}(z, y, x, a) f(z)\right)^{\prime}\right|<\frac{\alpha \pi}{2}
$$

$(z \in U ; 0<\alpha \leq 1)$ and

$$
\left|\arg \left(I_{\mu}^{\lambda}(z, y, x, a) g(w)\right)^{\prime}\right|<\frac{\alpha \pi}{2}
$$

$(w \in U ; 0<\alpha \leq 1)$ where the function is given by

$$
g(w)=w-a_{2} w^{2}+\left(a_{2}^{2}-a_{3}\right) w^{3}-\left(5 a_{2}^{3}-5 a_{2} a_{3}+a_{4}\right) w^{4}+\ldots
$$

We first state and prove the following result.
Theorem 1. Let $\mathrm{f}(\mathrm{z})$ given by (1) is said to be in the class $\mathscr{H}_{\Sigma}^{\alpha}$. Then

$$
\left|a_{2}\right| \leq \sqrt{\frac{2 \alpha^{2}}{2 p_{2}^{2}(1-\alpha)+3 p_{3} \alpha}} \text { and }\left|a_{3}\right|=\frac{\alpha^{2}}{p_{2}^{2} p_{3}}+\frac{2 \alpha}{3 p_{3}} .
$$

Proof. We can write the argument inequalities in (11) and (12) equivalently as follows:

$$
\begin{aligned}
& \left(I_{\mu}^{\lambda}(z, y, x, a) f(z)\right)^{\prime}=[Q(z)]^{\alpha} \quad \text { and } \\
& \left(I_{\mu}^{\lambda}(z, y, x, a) g(w)\right)^{\prime}=[L(w)]^{\alpha}
\end{aligned}
$$

respectively, where $Q(z)$ and $L(w)$ satisfy the following inequalities: $\mathfrak{R}(Q(z))>0,(z \in U)$ and $\mathfrak{R}(Q(z))>0,(w \in U)$. Furthermore, the functions $Q(z)$ and $L(w)$ have the forms

$$
\begin{gathered}
Q(z)=1+c_{1} z+c_{2} z^{2}+\ldots \quad \text { and } \\
L(w)=1+c_{1} w+c_{2} w^{2}+\ldots .
\end{gathered}
$$

Assume,

$$
\frac{(\lambda)_{n-1}}{(\mu)_{n-1} \Psi_{n-1}(y, x, a)}=p_{n},
$$

$$
\frac{(\lambda)_{1}}{(\mu)_{1} \Psi_{1}(y, x, a)}=p_{2}
$$

and

$$
\frac{(\lambda)_{2}}{(\mu)_{2} \Psi_{2}(y, x, a)}=p_{3}
$$

Then, $f(z)=z+\sum_{k=2}^{\infty} p_{n} a_{k} z^{k}$.

Now equating the coefficients of $\left(I_{\mu}^{\lambda}(z, y, x, a) f(z)\right)^{\prime}$ with $[Q(z)]^{\alpha}$ and the coefficients of $\left(I_{\mu}^{\lambda}(z, y, x, a) f(z)\right)^{\prime}$ with $[L(w)]^{\alpha}$, we get

$$
\begin{aligned}
2 p_{2} a_{2} & =\alpha c_{1} \\
3 p_{3} a_{3} & =\alpha c_{2}+\frac{\alpha(\alpha-1)}{2} c_{1}^{2} \\
-2 p_{2} a_{2} & =\alpha l_{1}
\end{aligned}
$$

and

$$
3 p_{3}\left(2 a_{2}^{2}-a_{3}\right)=\alpha l_{2}+\frac{\alpha(\alpha-1)}{2} l_{1}^{2}
$$

From (19) and (21), we get

$$
c_{1}=-l_{1} \text { and } 8 p_{2}^{2} a_{2}^{2}=\alpha^{2}+\left(c_{1}^{2}+l_{1}^{2}\right)
$$

Also, from (20) and (22), we find that

$$
6 p_{3} a_{2}^{2}-\left(\alpha c_{2}+\frac{\alpha(\alpha-1)}{2} c_{1}^{2}\right)=\alpha l_{2}+\frac{\alpha(\alpha-1)}{2} l_{1}^{2} \text {. }
$$

A rearrangement together with the second identity in (23) yields

$$
\begin{aligned}
6 p_{3} a_{2}^{2} & =\alpha\left(c_{2}+l_{2}\right)+\frac{\alpha(\alpha-1)}{2}\left(c_{1}^{2}+c_{1}^{2}\right) \\
& =\alpha\left(c_{2}+l_{2}\right)+\alpha(\alpha-1) \frac{4 p_{2}^{2} a_{2}^{2}}{\alpha^{2}}
\end{aligned}
$$

Therefore, we have 


$$
a_{2}^{2}=\frac{\alpha^{2}}{4 p_{2}^{2}(1-\alpha)+6 p_{3} \alpha}\left(c_{2}+l_{2}\right)
$$

which, in conjunction with the following well-known inequalities (see [1, p. 41]): $\left|c_{2}\right| \leq 2$ and $\left|l_{2}\right| \leq 2$, gives us the desired estimate on $\left|a_{2}\right|$ as asserted in (14).

Next, in order to find the bound on $\left|a_{3}\right|$, by subtracting (22) from (20), we get

$6 p_{3} a_{3}-6 p_{3} a_{2}^{2}=\alpha c_{2}+\frac{\alpha(\alpha-1)}{2} c_{1}^{2}-\left(\alpha l_{2}+\frac{\alpha(\alpha-1)}{2} l_{1}^{2}\right)$.

Upon substituting the value of $a_{2}^{2}$ from (23) and observing that $c_{1}^{2}=l_{1}^{2}$ it follows that

$$
a_{3}=\frac{\alpha^{2} c_{1}^{2}}{4 p_{2}^{2} p_{3}}+\frac{\alpha}{6 p_{3}}\left(c_{2}-l_{2}\right) .
$$

The familiar inequalities (see [1, p. 41]): $\left|c_{2}\right| \leq 2$ and $\left|l_{2}\right| \leq 2$, now yield

$$
\left|a_{3}\right|=\frac{\alpha^{2}}{p_{2}^{2} p_{3}}+\frac{2 \alpha}{3 p_{3}} .
$$

This completes the proof of theorem 1 .

\section{COEFFICIENT BOUNDS FOR THE CLASS $\mathscr{H}_{\Sigma}(\beta)$}

The Definition 1. A function $f(z)$ given by (1) is said to be in the class $\mathscr{H}_{\Sigma}(\beta) \quad(0 \leq \beta<1)$ if the following conditions are satisfied:

$$
f \in \sum \text { and } \quad \Re\left(I_{\mu}^{\lambda}(z, y, x, a) f(z)\right)^{\prime}>\beta,
$$

$(z \in U ; 0 \leq \beta<1)$ and

$$
\mathfrak{R}\left(I_{\mu}^{\lambda}(z, y, x, a) g(w)\right)^{\prime}>\beta,(w \in U ; 0 \leq \beta<1) .
$$

Theorem 2. Let $f(z)$ given by (1) is said to be in the class $\mathscr{H}_{\Sigma}(\beta) \quad(0 \leq \beta<1)$. Then

$$
\left|a_{2}^{2}\right| \leq \frac{2(1-\beta)}{3 p_{3}} \text { and }\left|a_{3}\right| \leq \frac{6 p_{3}}{p_{2}^{2}}(1-\beta)^{2}+4(1-\beta)
$$

where $p_{2}$ and $p_{3}$ in (17) and (18), respectively.

Proof. First of all, the argument inequalities in (24) and (25) can easily be rewritten in their equivalent forms:

$\left(I_{\mu}^{\lambda}(z, y, x, a) f(z)\right)^{\prime}=\beta+(1-\beta) Q(z)$

and

$\left(I_{\mu}^{\lambda}(z, y, x, a) g(w)\right)^{\prime}=\beta+(1-\beta) L(w)$

respectively, where $Q(z)$ and $L(w)$ satisfy the following inequalities: $\mathfrak{R}(Q(z))>0,(z \in U)$ and $\mathfrak{R}(Q(z))>0,(w \in U)$.

Moreover, the functions $Q(z)$ and $L(w)$ have the forms

$$
Q(z)=1+c_{1} z+c_{2} z^{2}+\ldots
$$

and

$$
L(w)=1+c_{1} w+c_{2} w^{2}+\ldots
$$

As in the proof of Theorem 1, by suitably comparing coefficients, we get

$$
\begin{aligned}
2 p_{2} a_{2} & =(1-\beta) c_{1} \\
3 p_{3} a_{3} & =(1-\beta) c_{2} \\
-2 p_{2} a_{2} & =(1-\beta) l_{1}
\end{aligned}
$$

and

$$
3 p_{3}\left(2 a_{2}^{2}-a_{3}\right)=(1-\beta) l_{2} \text {. }
$$

Now, considering (29) and (31)

$$
c_{1}=-l_{1} \text { and } 8 p_{2}^{2} a_{2}^{2}=(1-\beta)^{2}\left(c_{1}^{2}+l_{1}^{2}\right)
$$

Also, from (30) and (32), we find that

$$
6 p_{3} a_{2}^{2}=3 p_{3} a_{3}+(1-\beta) l_{2}=(1-\beta)\left(c_{2}+l_{2}\right) .
$$

Therefore, we have

$$
\left|a_{2}^{2}\right| \leq \frac{(1-\beta)}{6 p_{3}}\left(\left|c_{2}\right|+\left|l_{2}\right|\right)=\frac{2(1-\beta)}{3 p_{3}} .
$$

This gives the bound on $\left|a_{2}\right|$ as asserted in (26)

Next, in order to find the bound on $\left|a_{3}\right|$ by subtracting (33) and (30), we get

$$
6 p_{3} a_{3}-6 p_{3} a_{2}^{2}=(1-\beta)\left(c_{2}-l_{2}\right),
$$

which, upon substitution of the value of $a_{2}^{2}$ from (34), yields $6 p_{3} a_{3}=\frac{6 p_{3}}{8 p_{2}^{2}}(1-\beta)^{2}\left(c_{1}^{2}+l_{1}^{2}\right)+(1-\beta)\left(c_{2}+l_{2}\right)$.

This last equation, together with the well-known estimates: $\left|c_{1}\right| \leq 2,\left|l_{1}\right| \leq 2,\left|c_{2}\right| \leq 2$ and $\left|l_{2}\right| \leq 2$.

Lead us to the following inequality: $6 p_{3}\left|a_{3}\right| \leq \frac{3 p_{3}}{4 p_{2}^{2}}(1-\beta)^{2} .8+(1-\beta) .4$.

Therefore, we have $\left|a_{3}\right| \leq \frac{6 p_{3}}{p_{2}^{2}}(1-\beta)^{2}+4(1-\beta)$.

This completes the proof of Theorem 2 .

\section{REFERENCES}

[1] D. A. Brannan, J. Clunie (Eds). Aspect and Contemporary Complex Analysis (Proc. NATO Advanced Study Institute held at the University of Durham, Durham; July 1-20, 1979), Academic Press, New York and London, 1980.

[2] D. A. Brannan and T. S. Taha, On some classes of bi-univalen functions, in S.M. Mazhar, A. Hamoui, N.S. Faour (Eds.), Math. Anal. Appl., Kuwait; February 18-21, 1985, in KFAS Proceeding Series, vol. 3, Pergamon Press (Elsevier Science Limited), Oxpord, 1988, pp 53- 60; see also Studia Univ. Babes-Bolyai Math., 31(2), 70-77, 1986.

[3] E. Netanyahu, The minimal distance of the image boundary from the origin and the second coefficient of univalent function in $z<1$,Arch. Rational Mech. Anal. 32, 100-112, 1969.

[4] H. M. Srivastava, A. K. Misra and P. Gochhayat, Certain sublasses of analytic and bi-univalent functions, Apllied Math. Letters, 23, 11881192,2010

[5] M. Lewin, On a coefficient problem for bi-univalent functions, Proc. Amer. Math. Soc. 18, 63-68, 1967.

[6] T. S. Taha, Topics in Univalent Function Theory, Ph.D Thesis, University of London, 1981

[7] D. A. Brannan, J. Clunie, W. E. Kirwan, Coefficient estimates for a class of starlike functions, Canad. J. Math. 22, 476-485, 1970

[8] P.L. Duren, Univalent functions, in Grundlehren der Mathematischen Wissenschaften, Band 259, Springer-Verlag, New York Berlin, Heidelberg and Tokyo, 1983.

[9] R. W. Ibrahim and M. Darus, On operator by double Zeta functions, Tamkang J. Math., 42(2), 163-174, 2011.. 